\title{
THE DIRICHLET PROBLEM FOR RADIALLY HOMOGENEOUS ELLIPTIC OPERATORS
}

\author{
RICHARD F. BASS
}

\begin{abstract}
The Dirichlet problem in the unit ball is considered for the strictly elliptic operator $L=\sum a_{i j} D_{i j}$, where the $a_{i j}$ are smooth away from the origin and radially homogeneous: $a_{i j}(r x)=a_{i j}(x), r>0, x \neq 0$. Existence and uniqueness are proved for solutions in a certain space of functions. Necessary and sufficient conditions are given for an extended maximum principle to hold.
\end{abstract}

\section{INTRODUCTION}

Let $L$ be the second-order strictly elliptic operator defined by

$$
L f(x)=\sum_{i, j=1}^{d} a_{i j}(x) D_{i j} f(x),
$$

where the $a_{i j}$ are $C^{\infty}$ on $\mathbf{R}^{d}-\{0\}$ and radially homogeneous:

$$
a_{i j}(r x)=a_{i j}(x), \quad x \neq 0, r>0 .
$$

The main purpose of this paper is to investigate the Dirichlet problem in the unit ball for the operator $L$.

There is some question about what one means here by a solution to the Dirichlet problem. Of course, one does not expect the solution $h$ necessarily to be in $C^{2}$; one usually wants $h$ to be (locally) in the Sobolev space $W^{2, p}$ for some $p$. But a simple example of Pucci [11] shows that one cannot guarantee uniqueness of the solution, even with smooth boundary function, unless $p \geq d$, the dimension of the space. On the other hand, a recent example of Safonov [12] shows, among other things, that one may not have a solution at all unless $p \leq d / 2$. In fact, there is an example by Lamberti and Manselli [10] of an operator (although not a radially homogeneous one in a ball) where for each $p$ : either no solution exists in $W^{2, p}$ or else infinitely many solutions exist in $W^{2, p}$.

Received by the editors February 29, 1988 and, in revised form, October 10, 1988.

1980 Mathematics Subject Classification (1985 Revision). Primary 60J60; Secondary 35J15.

Key words and phrases. Dirichlet problem, maximum principle, elliptic operator, radially homogeneous, diffusion processes, martingale problem.

Research supported in part by NSF grant DMS-8701073. 
We formulate the Dirichlet problem slightly differently. Roughly speaking, we replace Lebesgue measure in the definition of $W^{2, p}$ with another measure that is naturally associated with the operator $L$. We consider the Dirichlet problem for boundary functions $f$ that are bounded and continuous on $\partial B_{1}$, the boundary of the open unit ball $B_{1}$. First of all, we require of any candidate for solution $h$ that

(i) $h$ is bounded and continuous on $\bar{B}_{1}$ (closure of $B_{1}$ );

(ii) $h$ is $C^{2}$ in $B_{1}-\{0\}$;

(iii) $L h \equiv 0$ in $B_{1}-\{0\}$; and

(iv) $h=f$ on $\partial B_{1}$.

We define $\Gamma(y)$ to be the Green function for $L$ for the unit ball with pole at 0 (a precise meaning of this is given in $\S 2$ ). We then prove (Theorem 2.3) that there exists $\eta>0$, depending only on the coefficients of $a$, with the property:

(1.2) There exists one and only one function $h$ satisfying (1.1) and

$$
\int_{B_{R}} \sum_{i, j=1}^{d}\left|D_{i j} h\right|^{1+\eta}(x) \Gamma(x) d x<\infty \text { for all } R<1 .
$$

Here $B_{R}$ is the open ball of radius $R$ about the origin.

Another main result of this paper concerns the extended maximal principle (cf. Gilbarg-Serrin [4]). We define a parameter $\bar{\mu}$ in terms of the $a_{i j}$. We then show (Theorem 2.1 and Proposition 2.2) that we have an extended maximum principle:

$$
\begin{aligned}
& \sup _{x \in B_{1}-\{0\}} h(x) \leq \sup _{x \in \partial B_{1}} h(x) \text { whenever } h \text { is bounded and } \\
& \text { continuous on } \bar{B}_{1}-\{0\} \text { and } L h \equiv 0 \text { on } B_{1}-\{0\} \text { if and only } \\
& \text { if } \bar{\mu} \geq 0 .
\end{aligned}
$$

As a by-product of our methods, we obtain the estimates

$$
\nabla h \in L^{d+\varepsilon}\left(B_{R}\right), \quad D_{i j} h \in L^{d / 2+\varepsilon}\left(B_{R}\right) \quad \text { for all } R<1 .
$$

The constant $\varepsilon$ depends only on the coefficients of ellipticity, and $\nabla h$ denotes the gradient of $h$. It would be interesting to know if the estimates (1.4) hold for nonradially homogeneous operators as well. Also, is the formulation of the Dirichlet problem given above, using (1.1) and (1.2), applicable more generally?

The approach taken in this paper is probabilistic, and one of our motivations was to compare solving the Dirichlet problem for $L$ to solving the corresponding martingale problem of probability theory. For the latter, the key step (see [1]) is to show that the largest eigenvalue $\alpha$ of a certain positive operator $Q$ is simple. For the Dirichlet problem more is required: we must also estimate hitting probabilities, Green functions, and rate of growth of solutions to $L h=0$ in terms of $\alpha$.

Concerning uniqueness (but not existence), there is a recent result of Caffarelli [15] that should be mentioned. Suppose the $a_{i j}$ are smooth except at 0 , 
strictly elliptic, but not necessarily radially homogeneous. Let $a_{i j}^{n}$ be smooth approximations to the $a_{i j}$ and let $h_{n}$ be the solution to the corresponding Dirichlet problem. Then Caffarelli showed that the functions $h_{n}$ converge, and the limit is independent of how the $a_{i j}$ were smoothed.

In $\S 2$ we give some preliminaries and state our results precisely. In $\S 3$ we give a criterion for whether the Markov process associated to $L$ ever hits the origin (nonpolar) or not (polar) and prove the extended maximum principle as a corollary. We then consider the more difficult of the two cases, the nonpolar one. In $\S 4$ we estimate hitting probabilities and the Green function, in $\S 5$ we establish existence of a solution to the Dirichlet problem, and in $\S 6$ we prove uniqueness of this solution. $\S 7$ covers the case where the origin is a polar set and the Markov process is transient, while $\S 8$ deals with the case where the origin is polar but the Markov process is neighborhood recurrent.

A few words about notation: We will use the letter $c$, with or without subscripts, to denote constants whose value is unimportant and may change from line to line. $B_{r}(x)$ denotes the open ball of radius $r$ about $x, B_{r}$ the open ball of radius $r$ about 0 .

\section{Preliminaries and Statement of Results}

Let the operator $L$ be defined by

$$
L f(x)=\sum_{i, j=1}^{d} a_{i j}(x) D_{i j} f(x),
$$

where the $a_{i j}$ satisfy

(i) (strict ellipticity) there exists $\kappa>0$ such that for all

$$
\begin{aligned}
& \left(y_{1}, \ldots, y_{d}\right) \in \mathbf{R}^{d}, x \in \mathbf{R}^{d}, \\
& \kappa \sum_{i=1}^{d} y_{i}^{2} \leq \sum_{i, j=1}^{d} y_{i} y_{j} a_{i j}(x) \leq \kappa^{-1} \sum_{i=1}^{d} y_{i}^{2} ;
\end{aligned}
$$

(ii) (smoothness) each $a_{i j}$ is $C^{\infty}$ on $\mathbf{R}^{d}-\{0\}$; and

(iii) (radial homogeneity) for each $i, j, a_{i j}(r x)=a_{i j}(x)$ whenever $r>0, x \neq 0$.

Let $S$ be the unit sphere. If we write $L$ in terms of polar coordinates $(r, \theta)$, $r>0, \theta \in S$, we get

$$
\begin{aligned}
L f(r, \theta)= & \frac{1}{2} \gamma(r, \theta) f_{r r}(r, \theta)+\gamma(r, \theta) \frac{2 \mu(r, \theta)+1}{2 r} f_{r}(r, \theta) \\
& +\frac{\gamma(r, \theta)}{r^{2}} L_{S} f(r, \theta)+\frac{\gamma(r, \theta)}{r} M f(r, \theta),
\end{aligned}
$$


where $f_{r}, f_{r r}$ are the derivatives of $f$ in the radial direction, $x=\left(x_{1}, \ldots, x_{d}\right)$ $=(r, \theta)$,

$$
\begin{aligned}
& \gamma(r, \theta)=2 r^{-2} \sum_{i, j=1}^{d} x_{i} a_{i j}(x) x_{j}, \\
& \mu(r, \theta)=\operatorname{trace}(a) / \gamma(r, \theta)-1,
\end{aligned}
$$

$L_{S}$ is an elliptic operator on $S$ containing the $f_{\theta}$ and $f_{\theta \theta}$ terms, and $M$ is an operator containing the mixed partials $f_{r \theta}$. The reason for the strange form of the coefficient of $f_{r}$ will be apparent shortly. By (2.2)(iii), $\gamma, \mu$, and the coefficients of $L_{S}$ and $M$ are independent of $r$, and we will write $\gamma(\theta), \mu(\theta)$. By (2.2)(i), $\gamma$ is bounded above and below away from 0. Hence using (2.2)(ii), $\gamma, \mu$, and the coefficients of $L_{S}$ and $M$ are $C^{\infty}$, and $L_{S}$ is strictly elliptic.

There is a unique strong Markov process $\left(P^{\theta}, \theta_{t}\right)$ with state space $S$ and infinitesimal generator $L_{S}$ (see [13]). Since $S$ is a compact manifold, $\left(P^{\theta}, \theta_{t}\right)$ has an invariant probability measure $\nu(d \theta)$ on $S$ [3, Example 3.1]. Equivalently, let $\nu(d \theta)$ be the measure with $\nu(S)=1$ whose density $v(\theta)$ with respect to surface measure is the nonnegative solution to $L_{S}^{*} v=0$, where $L_{S}^{*}$ is the adjoint operator to $L_{S}$.

Define

$$
\bar{\mu}=\int \mu(\theta) \nu(d \theta) .
$$

We then have the extended maximum principle:

Theorem 2.1. Suppose $\bar{\mu} \geq 0$. Suppose $h$ is bounded and continuous on $\bar{B}_{1}-$ $\{0\}, C^{2}$ in $B_{1}-\{0\}$, and satisfies $L h \equiv 0$ in $B_{1}-\{0\}$. Then

$$
\sup _{x \in B_{1}-\{0\}} h(x) \leq \sup _{x \in \partial B_{1}} h(x) .
$$

This is complemented by

Proposition 2.2. If $\bar{\mu}<0$, there exists $h$ bounded by 0 and 1 , continuous on $\bar{B}_{1}, C^{2}$ in $B_{1}-\{0\}$, and $L h \equiv 0$ in $B_{1}-\{0\}$ such that $h(0)=1$ and $h \equiv 0$ on $\partial B_{1}$.

Theorem 2.1 and Proposition 2.2 are proved in $\S 3$.

Consider the martingale problem for $L$ starting at $x$. This is the question of existence and uniqueness of a probability $P^{x}$ on the space of continuous paths in $\mathbf{R}^{d}$ such that

$$
P^{x}\left(X_{0}=x\right)=1
$$

and

$$
f\left(X_{t}\right)-f\left(X_{0}\right)-\int_{0}^{t} L f\left(X_{s}\right) d s \text { is a } P^{x} \text {-local martingale for each } f \in C^{2} .
$$


Existence follows by [13, Exercise 14.4.3]. Since the $a_{i j}$ are $C^{\infty}$ on $\mathbf{R}^{d}-\{0\}$, the proof that $P^{x}$ is determined up to the first time the process hits 0 is standard (see [13]). Uniqueness for the martingale problem then follows by [1] (although the context is slightly different, the proofs of $[1, \S 5]$ apply verbatim).

By Krylov [7], there exists a function $\Gamma(x)$, the Green function, such that

$$
E^{0} \int_{0}^{\tau_{1}} 1_{A}\left(X_{s}\right) d s=\int_{A} \Gamma(x) d x \text { for all } A \subseteq B_{1},
$$

where

$$
\tau_{r}=\inf \left\{t:\left|X_{t}\right|=r\right\} \text {. }
$$

One can show that $\Gamma$ is continuous away from 0 , but we do not need this.

Our formulation and solution to the Dirichlet problem is then given by

Theorem2.3. There exists $\eta>0$, depending only on $\kappa$, such that if $f$ is continuous on $\partial B_{1}$, then there exists one and only one function $h$ satisfying

(i) $h$ is bounded and continuous on $\bar{B}_{1}$;

(ii) $h$ is $C^{2}$ on $B_{1}-\{0\}$;

(iii) $L h=0$ on $B_{1}-\{0\}$;

(iv) $h$ agrees with $f$ on $\partial B_{1}$; and

(v) $\int_{B_{R}} \sum_{i, j=1}^{d}\left|D_{i j} h\right|^{1+\eta}(x) \Gamma(x) d x<\infty$ for all $R<1$.

For the case when $\bar{\mu} \geq 0$, it will turn out that (i)-(iv) are sufficient for uniqueness.

As a by-product of our proof of Theorem 2.3, we will get

Theorem 2.4. There exists $\varepsilon>0$, depending only on $\kappa$, such that if $h$ satisfies (2.8)(i)-(v), then

$$
\nabla h \in L^{d+\varepsilon}\left(B_{R}\right), \quad D_{i j} h \in L^{d / 2+\varepsilon}\left(B_{R}\right) \text { for all } R<1 .
$$

Theorems 2.3 and 2.4 will be proved in $\S 5$ (existence when $\bar{\mu}<0$ ), $\S 6$ (uniqueness when $\bar{\mu}<0$ ), $\S 7$ (the case $\bar{\mu}>0$ ), and $\S 8$ (the case $\bar{\mu}=0$ ).

Let $\widetilde{L}=\gamma(\theta)^{-1} L$. Note that $L h=0$ if and only if $\widetilde{L} h=0$. The Markov process corresponding to $\widetilde{L}$ may be obtained from that of $L$ by a time change, and it is well known (cf. $[13, \S 6.5]$ ) that if $\widetilde{\Gamma}$ is the corresponding Green function, $\widetilde{\Gamma}(x)=\gamma(\theta) \Gamma(x)$. Recall that $\gamma$ is bounded above and bounded below away from 0 . Thus, in the statements of Theorems 2.1, 2.3, and 2.4, there is no loss of generality in replacing $L$ by $\widetilde{L}$ or, equivalently, in assuming Assumption 2.5. $\sum_{i, j=1}^{d} x_{i} a_{i j}(x) x_{j} /|x|^{2}=\frac{1}{2}$ for all $x$.

Assumption 2.5 will remain in force for the remainder of the paper.

Writing $L$ in polar coordinates, we now get the much simpler expression

$$
L f(r, \theta)=\frac{1}{2} f_{r r}(r, \theta)+\frac{2 \mu(\theta)+1}{2 r} f_{r}(r, \theta)+\frac{1}{r^{2}} L_{S} f(r, \theta)+\frac{1}{r} M f(r, \theta),
$$


where

$$
\mu(\theta)=\operatorname{trace} a(x)-1, \quad x=(r, \theta) .
$$

We will use the following two lemmas frequently.

Lemma 2.6. Suppose $D$ is an open region in $\mathbf{R}^{d}, T=\inf \left\{t: X_{t} \notin D\right\}$, and $f$ is bounded on $\partial D$. Then $h(x)=E^{x} f\left(X_{T}\right)$ is continuous in $D$.

Proof. This follows by the proof of Theorem 2 in [8].

Lemma 2.7. Suppose $D, T, f$, and $h$ are as in Lemma 2.6. If $0 \notin D$, then $h$ is $C^{\infty}$ in $D$ and $L h=0$ there.

Proof. Fix $x_{0} \in D$ and choose $\varepsilon$ small enough so that $B_{2 \varepsilon}\left(x_{0}\right)$, the ball of radius $2 \varepsilon$ about $x_{0}$, lies in $D$. Let $S_{r}=\inf \left\{t: X_{t} \notin B_{r}\left(x_{0}\right)\right\}$. By the strong Markov property, $h(x)=E^{x} h\left(X_{S_{\varepsilon}}\right)$ if $x \in B_{\varepsilon}\left(x_{0}\right)$.

By Lemma 2.6, $h$ is continuous in $\overline{B_{\varepsilon}\left(x_{0}\right)}$. Let $u(x)$ be the solution to the Dirichlet problem for $L$ in $B_{\varepsilon}\left(x_{0}\right)$ with boundary function $h$. Since the $a_{i j}$ are uniformly $C^{\infty}$ on $B_{2 \varepsilon}\left(x_{0}\right)$, it is well known [5, Chapter 6] that $u$ is $C^{\infty}$ in $B_{\varepsilon}\left(x_{0}\right)$, continuous on $\overline{B_{\varepsilon}\left(x_{0}\right)}$, and $L u=0$ in $B_{\varepsilon}\left(x_{0}\right)$. By Ito's formula, $u\left(X_{t \wedge S_{r}}\right)$ is a martingale for $r<\varepsilon$. So

$$
u(x)=E^{x} u\left(X_{t \wedge S_{r}}\right), \quad r<\varepsilon .
$$

Using the boundedness of $u$ on $\overline{B_{\varepsilon}\left(x_{0}\right)}$, let $t \rightarrow \infty$, then $r \rightarrow \varepsilon$ to get

$$
u(x)=E^{x} u\left(X_{S_{\varepsilon}}\right)=E^{x} h\left(X_{S_{\varepsilon}}\right)=h(x) .
$$

Therefore $h(x)$ is $C^{\infty}$ and $L h=0$ in the interior of $B_{\varepsilon}\left(x_{0}\right)$. Since $x_{0}$ was arbitrary, this completes the proof.

\section{POLAR OR NONPOLAR}

Let $\left(P^{x}, X_{t}\right)$ be the unique solution to the martingale problem for $L$ starting at $x$. We will use repeatedly the fact that $\left(P^{x}, X_{t}\right)$ forms a strong Markov process $[13, \S 6.2]$.

In this section we give a criterion in terms of $\bar{\mu}$ for whether $X_{t}$ hits the origin in finite time or not. We use this to prove the extended maximum principle.

We begin by expressing $\left(P^{x}, X_{t}\right)$ in polar coordinates ( $\mathrm{cf}$. the skew product decomposition of [14]). Fix $x=(r, \theta)$, and write $X_{t}=\left(\widetilde{R}_{t}, \widetilde{\theta}_{t}\right)$. Recall that if $\sigma_{i j}(x)$ is a square root of $a_{i j}(x)$, we can write

$$
X_{t}^{(i)}=x^{(i)}+\int_{0}^{t} \sum_{j=1}^{d} \sigma_{i j}\left(X_{s}\right) d \widehat{W}_{x}^{(s)}, \quad i=1, \ldots, d,
$$

for some $d$-dimensional Brownian motion in $\widehat{W}$ (see [13]).

It follows by Ito's lemma, then, that $\widetilde{R}_{t}$ solves

$$
\widetilde{R}_{t}=r+\widetilde{W}_{t}+\int_{0}^{t}\left(2 \mu\left(\tilde{\theta}_{s}\right)+1\right) / 2 R_{s} d s
$$


up until the first time $\left|X_{t}\right|=0$ and that $\left(P^{(r, \theta)}, \tilde{\theta}_{t}\right)$ solves the martingale problem for $r^{-2} L_{S}$ starting at $(r, \theta)$. Here $\widetilde{W}_{t}$ is a standard one-dimensional Brownian motion.

Now let $\left(R_{t}, \theta_{t}\right)$ be the time change of $\left(\widetilde{R}_{t}, \tilde{\theta}_{t}\right)$ defined by

$$
R_{t}=\widetilde{R}\left(B_{t}^{-1}\right), \quad \theta_{t}=\tilde{\theta}\left(B_{t}^{-1}\right),
$$

where

$$
B_{t}=\int_{0}^{t} \widetilde{R}_{s}^{2} d s .
$$

Then, up until the first time of hitting $0, R_{t}$ solves

$$
d R_{t}=R_{t} d W_{t}+\left(\mu\left(\theta_{t}\right)+\frac{1}{2}\right) R_{t} d t, \quad R_{0}=r,
$$

where $W_{t}$ is a standard Brownian motion.

Let

$$
A_{t}=\int_{0}^{t} \mu\left(\theta_{t}\right) d s
$$

The equation (3.3) is linear in $R$, hence

$$
R_{t}=r \exp \left(W_{t}+A_{t}\right) .
$$

Also, note that $\left(P^{(r, \theta)}, \theta_{t}\right)$ solves the martingale problem for $L_{S}$ on $S$ starting at $\theta$. Since $L_{S}$ is smooth and strictly elliptic, $S$ is a smooth manifold, and the coefficients of $L_{S}$ depend only on $\theta$, there is at most one solution to the martingale problem for $L_{S}$ on $S$ starting at $\theta$. We denote it by $P^{\theta}$. By [13], $\left(P^{\theta}, \theta_{t}\right)$ forms a strong Markov process with state space $S$.

Theorem 3.1. For each $\theta$,

(a) if $\bar{\mu}>0, W_{t}+A_{t} \rightarrow+\infty, P^{\theta}$-a.s.;

(b) if $\bar{\mu}<0, W_{t}+A_{t} \rightarrow-\infty, P^{\theta}$-a.s.

Proof. $A_{t}$ is an additive functional of $\theta_{t}$, and by the ergodic theorem (see [2] or [3]), $A_{t} / t \rightarrow \bar{\mu}, P^{\theta}$-a.s.

Suppose $\bar{\mu}>0$. If $c>0$, it is well known that $W_{t}+c t \rightarrow+\infty$, a.s. as $t \rightarrow \infty$. Given $\varepsilon$, there exists $t_{0}$ such that

$$
P^{\theta}\left(A_{t} \leq \frac{1}{2} \bar{\mu} t \text { for some } t>t_{0}\right)<\varepsilon .
$$

Then for each $N$

$$
P^{\theta}\left(W_{t}+A_{t} \geq N \text { eventually }\right) \geq P^{\theta}\left(W_{t}+\frac{1}{2} \bar{\mu} t \geq N \text { eventually }\right)-\varepsilon \geq 1-\varepsilon,
$$
which proves $(\mathrm{a})$.

The proof of (b) is similar.

Let

$$
\tau_{r}=\inf \left\{t:\left|X_{t}\right|=r\right\} .
$$

We use Theorem 3.1 to get the criterion: 
Theorem 3.2. Suppose $x \neq 0$.

(a) If $\bar{\mu}>0, P^{x}\left(\tau_{0}<\infty\right)=0$ and $P^{x}\left(\left|X_{t}\right| \rightarrow \infty\right.$ as $\left.t \rightarrow \infty\right)=1$;

(b) if $\bar{\mu}<0, P^{x}\left(\tau_{0}<\infty\right)=0$ and $P^{x}\left(\left|X_{t}\right| \rightarrow \infty\right.$ as $\left.t \rightarrow \infty\right)=0$;

(c) if $\bar{\mu}=0, P^{x}\left(\tau_{0}<\infty\right)=0, P^{x}\left(\left|X_{t}\right| \rightarrow \infty\right)=0$, for all $r, P^{x}\left(\tau_{r}<\infty\right)=1$.

Proof. Write $x=(r, \theta)$. If $\bar{\mu}>0$, then $W_{t}+A_{t} \rightarrow+\infty$, a.s. by Theorem 3.1. Since $\log \left|R_{t}\right|=\log r+\left(W_{t}+A_{t}\right)$, then $\inf _{t} \log \left|R_{t}\right|>-\infty$, a.s. so $\tau_{0}=\infty$, a.s. and $\liminf \operatorname{in}_{t \rightarrow \infty} \log \left|R_{t}\right|=+\infty$, or $\left|R_{t}\right| \rightarrow \infty$ as $t \rightarrow \infty$. Part (a) follows easily.

Suppose now $\bar{\mu}<0$, and let $\varepsilon>0$. Since $W_{t}+A_{t} \rightarrow-\infty$, a.s., we can find $N_{0}$ such that for all $M$

$$
P^{\theta}\left(\log r+W_{t}+A_{t} \text { hits }-M \text { before hitting } N\right) \geq 1-\varepsilon
$$

whenever $N \geq N_{0}$. Since the time change that gives $\left|X_{t}\right|=\widetilde{R}_{t}$ from $R_{t}$ is nondegenerate (i.e., $d B_{t} / d t$ is bounded below) when $R_{t} \geq e^{-M}>0$, we have

$$
P^{x}\left(\left|X_{t}\right| \text { hits } e^{-M} \text { before hitting } e^{N}\right) \geq 1-\varepsilon \text {. }
$$

Let $M \rightarrow \infty$ to get $P^{x}\left(\tau_{0}<\tau_{\exp (N)}\right) \geq 1-\varepsilon$, then let $N \rightarrow \infty$, then $\varepsilon \rightarrow 0$. The second part of (b) follows from the first part and an elementary renewal argument.

Recall that $\left(P^{\theta}, \theta_{t}\right)$ is the process on $S$ with generator $L_{S}$. Since

$$
\int_{S} \mu(\theta) \nu(d \theta)=\bar{\mu}=0,
$$

it is well known (see [2]) that $E^{\theta} \mu\left(\theta_{t}\right)$ goes to 0 exponentially fast, uniformly in $\theta$, as $t \rightarrow \infty$.

Let

$$
d(\theta)=\int_{0}^{\infty} E^{\theta} \mu\left(\theta_{t}\right) d t
$$

Since $\mu(\theta)$ is $C^{\infty}$ and the coefficients of $L_{S}$ are $C^{\infty}$ and strictly elliptic, $d(\theta)$ is $C^{\infty}$ and

$$
L_{S} d=-\mu \text {. }
$$

Set

$$
u(x)=\log r+d(\theta), \quad x=(r, \theta) .
$$

Then applying (2.10), noting that the $u_{r \theta}$ terms are 0 , and using (3.8),

$$
L u(x)=0, \quad x \neq 0 .
$$

Hence $U_{t}=u\left(X_{t \wedge \tau_{0}}\right)$ is a local martingale.

By [1, Lemma 5.1], $P^{x}\left(\tau_{r}<\infty\right)=1$ if $|x|<r$. So $U_{t}$ is certain to exit the interval $[-M, N]$. Then, since $U_{t}$ is a time change of Brownian motion,

$$
P^{x}\left(U_{t} \text { hits }-M \text { before hitting } N\right)=(N-u(x)) /(M+N) \text {. }
$$


Using the definition of $u$, noting that $d(\theta)$ is bounded, and letting $M \rightarrow \infty$ leads as above to $P^{x}\left(\tau_{0}<\infty\right)=0$.

Similarly, holding $M$ fixed and letting $N \rightarrow \infty$ leads to $P^{x}\left(\tau_{r}<\infty\right)=1$ for all $r>0$ as above.

A renewal argument shows that $P^{x}\left(\left|X_{t}\right| \leq r\right.$ i.o. as $\left.t \rightarrow \infty\right)=1$, which implies $P^{x}\left(\left|X_{t}\right| \rightarrow \infty\right)=0$.

We refer to the cases where $\bar{\mu} \geq 0$ as polar since 0 is a polar set, the case $\bar{\mu}<0$ as nonpolar.

The proof of Theorem 2.1 is now easy.

Proof of Theorem 2.1. Suppose $\bar{\mu} \geq 0$, and suppose $h$ is bounded by $M$ on $\bar{B}_{1}-\{0\}$. Let $\gamma \in(0,1)$. By Lemma 2.7 , the solution to the Dirichlet problem in $B_{1}-B_{\gamma}$ with boundary values $h$ is again $h$, and if $|\gamma|<x$,

$$
\begin{aligned}
h(x) & =E^{x} h\left(X_{\tau_{1} \wedge \tau_{\gamma}}\right) \\
& =E^{x}\left(h\left(X_{\tau_{1}}\right) ; \tau_{1}<\tau_{\gamma}\right)+E^{x}\left(h\left(X_{\tau_{\gamma}}\right) ; \tau_{\gamma}<\tau_{1}\right) \\
& \leq \sup _{y \in \partial B_{1}} h(y)+M P^{x}\left(\tau_{\gamma}<\tau_{1}\right) .
\end{aligned}
$$

Let $\gamma \rightarrow 0$; by the continuity of paths, $\lim _{\gamma \rightarrow 0} P^{x}\left(\tau_{\gamma}<\tau_{1}\right)=P^{x}\left(\tau_{0}<\tau_{1}\right)=0$, using Theorem 3.2.

Proof of Proposition 2.2. Let $h(x)=P^{x}\left(\tau_{0}<\tau_{1}\right)$. By Lemmas 2.6 and 2.7, $h$ is $C^{2}$ in $B_{1}-\{0\}$ and $L h=0$ there. (Here $D=B_{1}-\{0\}, f(0)=1, f \equiv 0$ on $\partial B_{1}$.) It remains only to show (i) $\lim _{x \rightarrow 0} h(x)=1$ and (ii) $\lim _{x \rightarrow y} h(x)=0$ if $y \in \partial B_{1}$.

The first follows from scaling (see [1, proof of Proposition 5.3]): if $x=\varepsilon y$ wherc $|y|=1$, then

$$
P^{x}\left(\tau_{0}<\tau_{1}\right)=P^{y}\left(\tau_{0}<\tau_{1 / \varepsilon}\right) \rightarrow P^{y}\left(\tau_{0}<\tau_{\infty}\right)=1
$$

as $\varepsilon \rightarrow 0$.

For the second, by the strong Markov property, $h(x)=E^{x}\left(h\left(X_{\tau_{y}}\right) ; \tau_{\gamma}<\tau_{1}\right)$, where $\gamma=1 / 2<|x|$. The coefficients of $L$ are smooth on $B_{1}-B_{\gamma}$. So the solution to the classical Dirichlet problem for $L$ with boundary values $h$ on $\partial B_{\gamma}$ and 0 on $\partial B_{1}$ is continuous up to and including the boundary [5, Chapter 6]. By Lemma 2.7, the solution is $E^{x}\left(h\left(X_{\tau_{\gamma}}\right) ; \tau_{\gamma}<\tau_{1}\right)+E^{x}\left(0 ; \tau_{1}<\tau_{\gamma}\right)=h(x)$. Hence $h$ is continuous on $\partial B_{1}$.

\section{GREEN FUNCTION-NONPOLAR CASE}

In the next three sections we consider the nonpolar case: $\bar{\mu}<0$, which is the most difficult. First we introduce a positive operator $Q$, examine the eigenvalues of $Q$, and then estimate the probability of hitting 0 and estimate the Green function with pole at 0 in terms of the largest eigenvalue of $Q$. 
We suppose throughout this section that $\bar{\mu}<0$.

For $b \in(0,1)$, let $Q_{b}$ be the operator on functions on $S$ defined by

$$
Q_{b} f(x)=E^{b x}\left(f\left(X_{\tau_{1}}\right) ; \tau_{1}<\tau_{0}\right), \quad x \in S,
$$

where $\tau_{0}, \tau_{1}$ are defined by (3.6). We write simply $Q$ for $Q_{1 / 2}$.

Proposition 4.1. Let $b \in(0,1)$. Fix $x_{0} \in S$, and let $\pi(d y)=Q_{b}\left(x_{0}, d y\right)=$ $P^{b x_{0}}\left(X_{\tau_{1}} \in d y ; \tau_{1}<\tau_{0}\right)$. Then

(a) $Q_{b}$ is a bounded operator on $L^{2}(d \pi)$;

(b) $Q_{b}$ is a bounded operator from $L^{1}(d \pi)$ to $\mathscr{C}(S)$, the continuous functions on $S$;

(c) the largest eigenvalue $\lambda_{b}$ of $Q_{b}$ is positive and strictly larger in absolute value than any other eigenvalue of $Q_{b}, \lambda_{b}<1$, and the corresponding eigenfunction $e_{b}$ is continuous and strictly positive.

Proof. By the Harnack inequality of Krylov-Safonov [9], there exists a constant $\theta$ depending only on $\kappa$ of $(2.2)$ and $b$ such that if $f: S \rightarrow[0, \infty)$,

$$
\theta \leq \frac{E^{b x}\left(f\left(X_{\tau_{1}}\right) ; \tau_{1}<\tau_{0}\right)}{E^{b x_{0}}\left(f\left(X_{\tau_{1}}\right) ; \tau_{1}<\tau_{0}\right)} \leq \theta^{-1} \text { for all } x \in S .
$$

(Recall Lemma 2.7.) Consequently, $Q_{b}(x, d y)<<\pi(d y)$, and the density $q_{b}(x, y)=Q_{b}(x, d y) / \pi(d y)$ may be taken to be bounded by $\theta^{-1}$.

Since

$$
Q_{b} f(x)=\int f(y) Q_{b}(x, d y)=\int f(y) q_{b}(x, y) \pi(d y)
$$

and

$$
\int_{S} \int_{S} q_{b}(x, y)^{2} \pi(d x) \pi(d y) \leq \theta^{-2},
$$

(a) follows by Cauchy-Schwarz.

To prove (b), suppose $f \in L^{1}(d \pi)$ and $f \geq 0$. Then $Q_{b} f\left(x_{0}\right) \leq\|f\|_{L^{1}(d \pi)}$. By (4.2), $Q_{b} f(x) \leq \theta^{-1} Q_{b} f\left(x_{0}\right)$ for all $x \in S$, which proves $Q_{b} f$ is bounded on $S$.

By the Harnack inequality of Krylov-Safonov again, if $x \in S$ and $\varepsilon$ is taken small enough so that $B_{2 \varepsilon}(b x) \subseteq B_{1}(0)$, there exists a constant $c$ (depending on $\varepsilon$ and $\kappa)$ such that $h(y)=E^{y}\left(f\left(X_{\tau_{1}}\right) ; \tau_{1}<\tau_{0}\right) \leq c Q_{b} f(x)$ for $y \in B_{\varepsilon}(b x)$. By the strong Markov property,

$$
Q_{b} f(x)=E^{b x} h\left(X_{S_{\varepsilon}}\right)
$$

where

$$
S_{\varepsilon}=\inf \left\{t: X_{t} \notin B_{\varepsilon}\left(x_{0}\right)\right\} .
$$

So by Lemma 2.6, $Q_{b} f$ is continuous.

The operator $Q_{b}$ is strongly positive and compact; for a proof see [1, $\left.\S 5\right]$. Therefore, just as in the argument there, assertion (c), except for the $\lambda_{b}<1$ claim, follows by a the Krein-Rutman theorem. 
Pick $x$ such that $e_{b}(x) \equiv \sup _{y} e_{b}(y)$. Then

$$
\begin{aligned}
\lambda_{b} e_{b}(x) & =Q_{b} e_{b}(x)=E^{b x}\left(e_{b}\left(X_{\tau_{1}}\right) ; \tau_{1}<\tau_{0}\right) \leq \sup _{y} e_{b}(y) P^{b x}\left(\tau_{1}<\tau_{0}\right) \\
& =e_{b}(x) P^{b x}\left(\tau_{1}<\tau_{0}\right) .
\end{aligned}
$$

Since $e_{b}$ is strictly positive and $P^{b x}\left(\tau_{1}<\tau_{0}\right)<1$ by the proof of Theorem 3.3, $\lambda_{b} \leq P^{b x}\left(\tau_{1}<\tau_{0}\right)<1$.

We normalize $e_{b}$ so that $\int_{S} e_{b}^{2} d \pi=1$.

Although we know $\lambda_{b}>0$, we need for use in $\S 5$ to show that it is greater than a constant depending only on $\kappa$ and $b$.

Corollary 4.2. There exists a constant $c$ depending only on $\kappa$ and $b$ such that $\lambda_{b} \geq c$.

Proof. Analogously to the last paragraph of the proof of Proposition 4.1, $\lambda_{b} \geq$ $\inf _{x \in S} P^{b x}\left(\tau_{1}<\tau_{0}\right)$. But $P^{b x}\left(\tau_{1}<\tau_{0}\right)$ can be bounded below by a constant depending only on $\kappa$ and $b$ by the Stroock-Varadhan support theorem [6, Exercise 6.7.5] (cf. [1, proof of Theorem 5.4]).

Let

$$
\begin{aligned}
& d(\theta)=e_{1 / 2}(\theta), \quad \theta \in S, \\
& \alpha=\log \lambda_{1 / 2} / \log (1 / 2)>0,
\end{aligned}
$$

and let

$$
u(x)=r^{\alpha} d(\theta), \quad x=(r, \theta) .
$$

Proposition 4.3. (a) $u(x)=E^{x}\left(d\left(X_{\tau_{1}}\right) ; \tau_{1}<\tau_{0}\right), x \in B_{1}$;

(b) $u\left(X_{t \wedge \tau_{0}}\right)$ is a $P^{x}$-local martingale for all $x$;

(c) $d$ is bounded above and bounded below away from $0 ; d$ is $C^{\infty}$ on $S$. Proof. Suppose $b \in(0,1)$. By the strong Markov property and radial homogeneity, $\left(Q_{b}\right)^{n}=Q_{b^{n}}$, and so $Q_{b^{n}} e_{b}=\lambda_{b}^{n} e_{b}$. By the Krein-Rutman theorem [6, Theorem 6.3], $Q_{b^{n}}$ has only one strictly positive eigenfunction, hence $e_{b^{n}}$ is a constant multiple of $e_{b}$, and $\lambda_{b^{n}}=\left(\lambda_{b}\right)^{n}$.

Now suppose $|x|=(1 / 2)^{m / n}, m, n$ positive integers, and let $b=|x|$. $Q_{b^{n}}=Q_{(1 / 2)^{m}}$, hence $\lambda_{b}^{n}=\lambda_{1 / 2}^{m}$, and $Q_{b} d=\lambda_{b} d$. Therefore

$$
\begin{aligned}
E^{x}\left(d\left(X_{\tau_{1}}\right) ; \tau_{0}<\tau_{0}\right) & =Q_{b} d(x / b)=\lambda_{b} d(x / b)=\left(\lambda_{1 / 2}\right)^{m / n} d(x /|x|) \\
& =|x|^{\alpha} d(x /|x|) .
\end{aligned}
$$

This proves (a) for $|x|$ of the form $(1 / 2)^{s}, s$ rational. $E^{x}\left(d\left(X_{\tau_{1}}\right) ; \tau_{1}<\tau_{0}\right)$ is continuous in $B_{1}-\{0\}$ by Lemma 2.6 , and $u$ is evidently continuous as a function of $|x|$. So (a) holds for $x \in B_{1}-\{0\}$. 
Now

$$
\left|E^{x}\left(d\left(X_{\tau_{1}}\right) ; \tau_{1}<\tau_{0}\right)\right| \leq\|d\| P^{x}\left(\tau_{1}<\tau_{0}\right) \rightarrow 0 \text { as } x \rightarrow 0
$$

by the argument in the proof of Proposition 2.2. So (a) holds for $x=0$.

By Lemma 2.7, $u(x)$ is $C^{\infty}$ in $B_{1}-\{0\}$ and $L u=0$ there. So

$$
0=L u=r^{\alpha-2}\left[\alpha(\alpha-1) d / 2+\alpha(2 \mu+1) d / 2+L_{S} d+\alpha M(r d)\right]
$$

for $r \in(0,1)$. But then the expression inside the brackets, which is independent of $r$, is 0 for all $\theta$, hence (4.6) holds for all $r>0$. (b) then follows by Ito's lemma.

We have already mentioned that $u$ is $C^{\infty}$ in $B_{1}-\{0\}$. This shows $d$ is smooth. The boundedness part of (c) follows immediately from Proposition 4.1(c).

Corollary 4.4. $d(\theta)$ is bounded above and below by constants depending only on $\kappa$. There is a modulus of continuity for $d(\theta)$ depending only on $\kappa$.

Proof. Since $d=e_{1 / 2}$ is normalized to have $L^{2}$ norm one with respect to a certain measure, there is at least one point $x$ for which $d(x) \leq 1$. Hence $Q d(x) \leq \lambda_{1 / 2}$, and by (4.2),

$$
\sup _{y \in S} d(y)=\left(\lambda_{1 / 2}\right)^{-1} \sup _{y \in S} Q d(y) \leq\left(\lambda_{1 / 2}\right)^{-1} \theta^{-1} Q d(x) \leq \theta^{-1} .
$$

Since there must be at least one point at which $d$ is $\geq 1$, the lower bound is similar. The assertion about the modulus of continuity follows from Lemma 2.6 and its proof.

The $Q=Q_{1 / 2}$ that we have defined here is, by scaling, the same as the $Q$ defined in [1]. Williams [14] has given a quite different method of constructing a positive solution to $L u=0$. Our construction sheds additional light on hers, and vice versa.

Having constructed the function $u$, we can now begin doing some estimation. Let

$$
\begin{gathered}
V_{i}=\left\{x: u(x) \leq 2^{i}\right\}, \quad i=\ldots,-2,-1,0,1,2, \ldots, \\
F_{i}=\partial V_{i},
\end{gathered}
$$

and

$$
\sigma_{i}=\inf \left\{t: X_{t} \in F_{i}\right\} .
$$

Proposition 4.5. There exist constants $c_{1}, c_{2}>0$ such that

$$
c_{1}|x|^{\alpha} \leq P^{x}\left(\tau_{1}<\tau_{0}\right) \leq c_{2}|x|^{\alpha}
$$

for $x$ sufficiently small.

Proof. Since $d(\theta)$ is bounded below there exists $i_{2}$ such that $V_{i_{2}} \supseteq B_{1}$. Then

$$
\begin{aligned}
P^{x}\left(\tau_{1}<\tau_{0}\right) & \geq P^{x}\left(\sigma_{i_{2}}<\tau_{0}\right)=P^{x}\left(u\left(X_{t}\right) \text { hits } 2^{i_{2}} \text { before hitting } 0\right) \\
& =u(x) / 2^{i_{2}} \geq c_{1}|x|^{\alpha}
\end{aligned}
$$

since $u\left(X_{t}\right)$ is a martingale. 
Similarly, there exists $c_{1}$ such that $V_{i_{1}} \subseteq B_{1}$, and then

$$
\begin{aligned}
P^{x}\left(\tau_{1}<\tau_{0}\right) & \leq P^{x}\left(\sigma_{i_{1}}<\tau_{0}\right)=P^{x}\left(u\left(X_{t}\right) \text { hits } 2^{i_{1}} \text { before hitting } 0\right) \\
& =u(x) / 2^{i_{1}} \leq c_{2}|x|^{\alpha} .
\end{aligned}
$$

As a corollary, we have

$$
\lim _{|x| \rightarrow 0}\left(\log P^{x}\left(\tau_{1}<\tau_{0}\right) / \log |x|\right)=\alpha .
$$

On the other hand, it is possible to estimate $P^{x}\left(\tau_{1}<\tau_{0}\right)$ in terms of the probability that $\log |x|+W_{t}+A_{t}$ ever hits 0 (see $\S 3$ ). Although this does not quite fit into standard large-deviations theory, one might be able to get asymptotic estimates by techniques from that theory; tying $\mu(\theta)$ and $\alpha$ together would answer some questions raised by Williams [14] in a similar context.

Lemma 4.6. $\alpha \leq 1$.

Proof. From (2.10) and Assumption 2.5, $\mu(\theta) \geq-\frac{1}{2}$.

From (2.10) and Ito's formula, $\left|X_{t}\right|$ is a submartingale whose martingale part is a Brownian motion, $\widehat{W}_{t}$. Hence, for $x$ small,

$$
P^{x}\left(\tau_{1}<\tau_{0}\right) \geq P^{0}\left(\widehat{W}_{t}+|x| \text { hits } 1 \text { before hitting } 0\right)=|x| .
$$

By Proposition 4.5,

$$
c_{2}|x|^{\alpha} \geq|x|
$$

for all $|x|$ sufficiently small, which implies $\alpha \leq 1$.

We now come to the main estimate of this section.

Theorem 4.7. There exist constants $c_{1}$ and $c_{2}$ such that

$$
\Gamma(x) \leq c_{2}|x|^{2-d-\alpha} \quad \text { a.e. on } B_{1}
$$

and

Proof. Let

$$
\Gamma(x) \geq c_{1}|x|^{2-d-\alpha} \quad \text { a.e. on some neighborhood of } 0 .
$$

$$
\begin{gathered}
A_{i}=V_{i+4}-V_{i-3}, \\
G_{i}=\sup \left\{g_{A_{i}}(x, y): x \in F_{i-2} \cup F_{i+3}, y \in V_{i+2}-V_{i-1}\right\},
\end{gathered}
$$

where $g_{A_{i}}$ is the Green function for $A_{i}$ with pole at $x$. Since $L$ is smooth on $V_{i-3}^{c}, g_{A_{i}}$ is continuous in $x$ and $y$ except at $x=y$. Hence $G_{i}<\infty$.

Suppose $y \in V_{i+2}-V_{i-1}$ and let $B_{\varepsilon}(y)$ be the ball of radius $\varepsilon$ about $y$, $\varepsilon$ sufficiently small so that $B_{2 \varepsilon}(y) \subseteq V_{i+3}-V_{i-1}$. We will write $g_{A_{i}}\left(x, B_{\varepsilon}(y)\right)$ for $\int_{B_{\varepsilon}(y)} g_{A_{i}}(x, z) d z$. Using scaling with the factor $2^{-1 / \alpha}$ (cf. [1, Proposition 5.3]), the expected amount of time spent in $B_{\varepsilon}(y)$ before leaving $A_{i}$ starting at 
$x$ is $2^{-2 / \alpha}$ times the expected amount of time spent in $B_{2^{1 / \alpha} \varepsilon}\left(2^{1 / \alpha} y\right)$ starting at $2^{1 / \alpha} x$ before leaving $A_{i+1}$, or

$$
g_{A_{i}}\left(x, B_{\varepsilon}(y)\right)=2^{-2 / \alpha} g_{A_{i+1}}\left(2^{1 / \alpha} x, B_{2^{1 / \alpha} \varepsilon}\left(2^{1 / \alpha} y\right)\right) .
$$

Dividing by the volume of $B_{\varepsilon}(y)$, letting $\varepsilon \rightarrow 0$, and using the continuity at $y$,

$$
g_{A_{i}}(x, y)=2^{-2 / \alpha} 2^{d / \alpha} g_{A_{i+1}}\left(2^{1 / \alpha} x, 2^{1 / \alpha} y\right)
$$

Hence

$$
G_{i}=2^{(d-2) / \alpha} G_{i+1} \text {. }
$$

Fixing $i_{2}$ so that $B_{1} \subseteq V_{i_{2}-5}$ and then induction gives

$$
G_{i}=2^{(d-2)\left(i_{2}-i\right) / \alpha} G_{i_{2}} \leq c 2^{(2-d) i / \alpha} .
$$

Now let

$$
H_{i}^{\varepsilon}=\sup \left\{g\left(x, B_{\varepsilon}(y)\right): x \in F_{i-2} \cup F_{i+3}, y \in V_{i+1}-V_{i}\right\},
$$

where

$$
g\left(x, B_{\varepsilon}(y)\right)=E^{x} \int_{0}^{\sigma_{i_{2}}} 1_{B_{\varepsilon}(y)}\left(X_{s}\right) d s,
$$

$\sigma_{i_{2}}$ is defined by (4.9), and $B_{2 \varepsilon}(y) \subseteq V_{i+2}-V_{i-1}$ for all $y \in V_{i+1}-V_{i}$.

Fix $y \in V_{i+1}-V_{i}, i<i_{2}-5$. Starting at $z \in F_{i-3}$, one is certain to hit $F_{i-2}$ before entering $B_{\varepsilon}(y)$, and so by the strong Markov property, $g\left(z, B_{\varepsilon}(y)\right) \leq$ $H_{i}^{\varepsilon}$.

If $z \in F_{i+4}$,

$$
\begin{aligned}
P^{z}\left(\sigma_{i+3}<\sigma_{i_{2}}\right) & =P^{z}\left(u\left(X_{t}\right) \text { hits } 2^{i+3} \text { before hitting } 2^{i_{2}}\right) \\
& =\left(2^{i_{2}}-2^{i+4}\right) /\left(2^{i_{2}}-2^{i+3}\right) \leq 1-c 2^{i} .
\end{aligned}
$$

So by the strong Markov property, $g\left(z, B_{\varepsilon}(y)\right) \leq\left(1-c 2^{i}\right) H_{i}^{\varepsilon}$ if $z \in F_{i+4}$.

Let $T_{i}=\inf \left\{t: X_{t} \notin A_{i}\right\}$. Then just as in (4.16),

$$
P^{x}\left(X_{T_{i}} \in F_{i+4}\right)= \begin{cases}\left(2^{7}-1\right)^{-1}, & x \in F_{i-2}, \\ \left(2^{6}-1\right) /\left(2^{7}-1\right), & x \in F_{i+3} .\end{cases}
$$

Using the strong Markov property again and (4.17), if $x \in F_{i-2} \cup F_{i+3}$,

$$
\begin{aligned}
g\left(x, B_{\varepsilon}(y)\right)= & g_{A_{i}}\left(x, B_{\varepsilon}(y)\right)+E^{x} g\left(X_{T_{i}}, B_{\varepsilon}(y)\right) \\
\leq & g_{A_{i}}\left(x, B_{\varepsilon}(y)\right)+H_{i}^{\varepsilon} P^{x}\left(X_{T_{i}} \in F_{i-3}\right) \\
& +\left(1-c 2^{i}\right) H_{i}^{\varepsilon} P^{x}\left(X_{T_{i}} \in F_{i+4}\right) \\
\leq & \omega_{d} \varepsilon^{d} G_{i}+H_{i}^{\varepsilon}\left(1-c 2^{i}\right) .
\end{aligned}
$$

Here $\omega_{d}$ denotes the Lebesgue measure of the unit ball. 
Holding $i$ fixed but taking the sup over $y \in V_{i+1}-V_{i}, x \in F_{i-2} \cup F_{i+3}$,

$$
H_{i}^{\varepsilon} \leq \omega_{d} \varepsilon^{d} G_{i}+H_{i}^{\varepsilon}\left(1-c 2^{i}\right)
$$

or, from (4.14),

$$
H_{i}^{\varepsilon} \leq c 2^{-i} G_{i} \omega_{d} \varepsilon^{d} \leq c 2^{i(2-d-\alpha) / \alpha} \omega_{d} \varepsilon^{d} .
$$

Since $\sigma_{i_{2}}>\tau_{1}$ and $|y|^{\alpha} \leq c u(y) \leq c 2^{i+1}$ if $y \in V_{i+1}-V_{i}$, the strong Markov property yields

$$
\int_{B_{\varepsilon}(y)} \Gamma(z) d z \leq g\left(0, B_{\varepsilon}(y)\right) \leq H_{i}^{\varepsilon} \leq c|y|^{2-d-\alpha} \omega_{d} \varepsilon^{d} .
$$

Since $\varepsilon$ can be arbitrarily small, this yields the upper bound.

Choose $i_{1}$ so that $V_{i_{1}+5} \subseteq B_{1}$. Replacing $i_{2}$ by $i_{1}$, replacing sup by inf in the definitions of $G_{i}$ and $H_{i}^{\varepsilon}$, noting $G_{i}>0$, and reversing the inequalities in the above argument gives the lower bound. However, the argument is valid only for $y \in V_{i_{1}}$, which is why the lower bound holds only for a neighborhood of 0 .

\section{EXISTENCE-NONPOLAR CASE}

In this section we establish the existence part of Theorem 2.3 in the case $\bar{\mu}<0$. Suppose $f$ is bounded and continuous on $\partial B_{1}$.

Let

$$
h(x)=E^{x} f\left(X_{\tau_{1}}\right) .
$$

Recall the definition of $V_{i}, F_{i}$, and $\sigma_{i}$ in (4.7)-(4.9). Write $\operatorname{Osc}_{A} h$ for $\sup _{A} h-\inf _{A} h$.

Proposition 5.1. There exists $\gamma<1$ depending only on $\kappa$ such that

$$
\underset{V_{j}}{\operatorname{Osc}} h \leq \frac{1}{2} \gamma \underset{V_{j+1}}{\operatorname{Osc} h}, \quad \text { provided } V_{j+1} \subseteq B_{1} .
$$

Proof. By taking a linear transformation, we may suppose that $\inf _{V_{j+1}} h=0$ and $\sup _{V_{j+1}} h=1$. Since $h$ is continuous in $\bar{V}_{j+1}$ by Lemma 2.6 and $h(x)=$ $E^{x} h\left(X_{\sigma_{j+1}}\right)$ for $x \in V_{j+1}$ by the strong Markov property, we have $\inf _{F_{j+1}} h=0$, $\sup _{F_{j+1}} h=1$.

Since $h(x)=E^{x} h\left(X_{\sigma_{j}}\right)$ for $x \in V_{j}, \operatorname{Osc}_{V_{j}} h \leq \operatorname{Osc}_{F_{j}} h$, and the proposition will be proved if we show

$$
\underset{F_{j}}{\mathrm{Osc}} h \leq \frac{1}{2} \gamma \underset{F_{j+1}}{\operatorname{Osc}} h .
$$

Fix $j_{0}$. In view of Corollaries 4.2 and 4.4 and the definition of $u$, the minimum distance between $F_{j_{0}}$ and $F_{j_{0}+1}$ is bounded below by a constant 
depending only on $\kappa$. So by the Harnack inequality of Krylov-Safonov [9], there exists $\theta$ depending only on $\kappa$ such that for $j=j_{0}$,

$$
\theta \leq \frac{P^{x}\left(X_{\sigma_{j+1}} \in A ; \sigma_{j+1}<\tau_{0}\right)}{P^{y}\left(X_{\sigma_{j+1}} \in A ; \sigma_{j+1}<\tau_{0}\right)} \leq \theta^{-1}, \quad x, y \in F_{j}, A \subseteq F_{j+1} .
$$

By scaling, (5.3) holds for all $j$, with $\theta$ independent of $j$.

Fix $x_{0} \in F_{j}$. By looking at $1-h$ if necessary, we may suppose

$$
P^{x_{0}}\left(X_{\sigma_{j+1}} \in A^{+} ; \sigma_{j+1}<\tau_{0}\right) \geq P^{x_{0}}\left(X_{\sigma_{j+1}} \in A^{-} ; \sigma_{j+1}<\tau_{0}\right),
$$

where

$$
A^{+}=\left\{y \in F_{j+1}: h(y) \geq \frac{1}{2}\right\}, \quad A^{-}=\left\{y \in F_{j+1}: h(y) \leq \frac{1}{2}\right\} .
$$
So

For any $x \in F_{j}, P^{x}\left(\tau_{0}<\sigma_{j+1}\right)=P^{x}\left(u\left(X_{t}\right)\right.$ hits 0 before hitting $\left.2^{j+1}\right)=\frac{1}{2}$.

$$
\begin{aligned}
h(x) & =E^{x} h\left(X_{\sigma_{j+1}}\right)=E^{x}\left(h\left(X_{\sigma_{j+1} \wedge \tau_{0}}\right) ; \sigma_{j+1}<\tau_{0}\right)+h(0) P^{x}\left(\tau_{0}<\sigma_{j+1}\right) \\
& \leq P^{x}\left(\sigma_{j+1}<\tau_{0}\right)+h(0) P^{x}\left(\tau_{0}<\sigma_{j+1}\right) \leq \frac{1}{2}+\frac{1}{2} h(0) .
\end{aligned}
$$

But also,

$$
\begin{aligned}
h(x) & =E^{x}\left(h\left(X_{\sigma_{j+1}}\right) ; \sigma_{j+1}<\tau_{0}\right)+\frac{1}{2} h(0) \\
& \geq \frac{1}{2} P^{x}\left(X_{\sigma_{j+1}} \in A^{+} ; \sigma_{j+1}<\tau_{0}\right)+\frac{1}{2} h(0) \\
& \geq \frac{1}{2} \theta P^{x_{0}}\left(X_{\sigma_{j+1}} \in A^{+} ; \sigma_{j+1}<\tau_{0}\right)+\frac{1}{2} h(0) \\
& \geq \frac{1}{4} \theta P^{x_{0}}\left(\sigma_{j+1}<\tau_{0}\right)+\frac{1}{2} h(0) \\
& =\frac{1}{8} \theta+\frac{1}{2} h(0) .
\end{aligned}
$$

Comparing (5.5) and (5.6), we have

$$
\underset{F_{j}}{\operatorname{Osc}} h \leq \frac{1}{2}-\frac{1}{8} \theta=\frac{1}{2}\left(1-\frac{1}{4} \theta\right),
$$

which gives (5.2) with $\gamma=1-\frac{1}{4} \theta$.

Proposition 5.3. There exists $\delta_{1}>0$ depending only on $\kappa$ such that

$$
\underset{B_{r}}{\operatorname{Osc}} h \leq c r^{\alpha\left(1+\delta_{1}\right)}\|f\|, \quad r<1 .
$$

Proof. Pick $i_{1}$ so that $V_{i_{1}} \subseteq B_{1}$. Suppose $r$ is sufficiently small of that $B_{r} \subseteq$ $V_{i_{1}}$. Given $r$, let $j$ be the smallest integer such that $B_{r} \subseteq V_{j}$. Then $2^{j} \leq c|r|^{\alpha}$ and

$$
\begin{aligned}
\underset{B_{r}}{\operatorname{Osc}} h & \leq \underset{V_{j}}{\operatorname{Osc}} h \leq\left(\frac{1}{2} \gamma\right)^{i_{1}-j} \underset{V_{i_{1}}}{\operatorname{Osc}} h \leq c\left(\frac{1}{2} \gamma\right)^{-j} \underset{B_{1}}{\operatorname{Osc}} h \\
& \leq c r^{\alpha\left(1+\delta_{1}\right)} \underset{B_{1}}{\operatorname{Osc}} h,
\end{aligned}
$$

where $\delta_{1}=-\ln \gamma / \ln 2$. 
Since $\operatorname{Osc}_{B_{1}} h \leq 2\|f\|$ follows from (5.1), this proves the proposition for $r$ sufficiently small. Since $\operatorname{Osc}_{B_{r}} h \leq \operatorname{Osc}_{B_{1}} h \leq 2\|f\|$, by taking $c$ larger if necessary, we have the proposition for all $r<1$.

Proposition 5.3. There exists $\delta_{2}>0$ depending only on $\kappa$ such that

$$
\underset{B_{r}}{\operatorname{Osc}} h \leq c r^{\delta_{2}}\|f\|, \quad r<1 .
$$

Proof. This is similar to the preceding, but a little simpler. By the Harnack inequality, pick $\theta^{\prime}$ such that

$$
\theta^{\prime} \leq \frac{P^{x}\left(X_{\tau_{1}} \in A\right)}{P^{y}\left(X_{\tau_{1}} \in A\right)} \leq\left(\theta^{\prime}\right)^{-1}, \quad x, y \in \partial B_{1 / 2}, A \subseteq \partial B_{1} .
$$

Fix $x_{0} \in \partial B_{r}, r \leq \frac{1}{2}$, and suppose $\sup _{\partial B_{2 r}} h=1, \inf _{\partial B_{2 r}} h=0$, and

$$
P^{x_{0}}\left(X_{\tau_{2 r}} \in A^{+}\right) \geq P^{x_{0}}\left(X_{\tau_{2 r}} \in A^{-}\right)
$$

with

$$
A^{+}=\left\{y \in \partial B_{2 r}: h(y) \geq \frac{1}{2}\right\}, \quad A^{-}=\left\{y \in \partial B_{2 r}: h(y) \leq \frac{1}{2}\right\} .
$$

Then if $x \in \partial B_{r}, h(x) \leq 1$, and by (5.7) and scaling,

$$
\begin{aligned}
h(x) & =E^{x} h\left(X_{\tau_{2 r}}\right) \geq \frac{1}{2} P^{x}\left(X_{\tau_{2 r}} \in A^{+}\right) \\
& \geq \frac{1}{2} \theta^{\prime} P^{x_{0}}\left(X_{\tau_{2 r}} \in A^{+}\right) \geq \frac{1}{4} \theta^{\prime} .
\end{aligned}
$$

Hence

$$
\underset{B_{r}}{\operatorname{Osc}} h \leq\left(1-\frac{1}{4} \theta^{\prime}\right) \underset{B_{2 r}}{\operatorname{Osc}} h, \quad r \leq \frac{1}{2} .
$$

Proposition 5.3 follows from (5.8) similarly to the proof of Proposition 5.2 .

Corollary 5.4. There exists $\delta$ depending only on $\kappa$ such that

$$
\underset{B_{r}}{\operatorname{Osc}} h \leq c r^{\alpha+\delta}\|f\| \text {. }
$$

Proof. Let $\delta=\left(1 \wedge \delta_{1}\right) \delta_{2} / 2$. If $\alpha<\delta_{2} / 2$, the corollary follows by Proposition 5.3. If $\alpha \geq \delta_{2} / 2$, it follows by Proposition 5.2.

Theorem 5.5. $h(x)$ defined by (5.1) satisfies (2.8)(i)-(v).

Proof. The boundedness of $h$ is clear by (5.1). Assertions (ii) and (iii) follow by Lemma 2.7. Since $h$ agrees with the unique solution to the Dirichlet problem for $L$ on $B_{1}-B_{1 / 2}$ with boundary function $f$ on $\partial B_{1}$ and $h$ on $\partial B_{1 / 2}, h$ is continuous at $\partial B_{1}$ and agrees with $f$ there (cf. proof of Lemma 2.7). The continuity of $h$ at 0 comes from Lemma 2.6, and it remains to prove (v).

By the bounds on $\Gamma$ from Theorem 4.7 and the fact that $h \in C^{2}$ on $B_{1}-\{0\}$, it suffices to restrict attention to $R<1 / 2$. By adding or subtracting a constant, we may suppose $h(0)=0$. Then if $x \in B_{R}, h$ solves the Dirichlet problem for 
$L$ on the ball $B_{|x| / 2}(x)$ with boundary function $h$ by Lemma 2.7. So $h \in C^{2}$ there, and it is well known [5, Chapter 6] that

$$
\left|D_{i j} h(y)\right| \leq c|x|^{-2} \sup _{z \in \partial B_{|x| / 2}(x)}|h(z)|, \quad y \in B_{|x| / 4}(x) .
$$

The constant $c$ may depend on the smoothness of the $a_{i j}$.

But then

$$
\left|D_{i j} h(x)\right| \leq c|x|^{-2} \sup _{z \in B_{3|x| / 2}}|h(x)| \leq c|x|^{-2} \operatorname{Osc}_{B_{3|x| / 2}} h \leq c|x|^{-2+\alpha+\delta},
$$

by Corollary 5.4 and the fact that $h(0)=0$.

Estimate (5.10) and Theorem 4.7 give, changing to polar coordinates,

$$
\int_{B_{R}}\left|D_{i j} h(x)\right|^{1+\eta} \Gamma(x) d x \leq c \int_{0}^{R} r^{d-1}\left(r^{-2+\alpha+\delta}\right)^{1+\eta} r^{2-d-\alpha} d r .
$$

Recalling Lemma 4.6, this gives (v) if $\eta<\delta /(2-\alpha-\delta)$.

Corollary 5.6. There exists $\varepsilon>0$ depending only on $\kappa$ such that

$$
\nabla h \in L^{d+\varepsilon}\left(B_{R}\right), \quad D_{i j} h \in L^{d / 2+\varepsilon}\left(B_{R}\right), \quad R<1 .
$$

Proof. The second assertion follows by integrating estimate (5.10) in polar coordinates. Just as we obtained (5.10), we get

$$
\left|D_{i} h(x)\right| \leq c|x|^{-1} \sup _{z \in B_{3|x| / 2}}|h(z)| \leq c|x|^{-1+\alpha+\delta} .
$$

Integrate (5.11) in polar coordinates to get the first assertion.

\section{UNIQUENESS-NONPOLAR CASE}

In this section we complete the proof of Theorem 2.3 for the case $\bar{\mu}<0$ by showing uniqueness. We first show uniqueness for the Dirichlet problem for $V_{i_{1}}$, where $i_{1}$ is chosen so that $V_{i_{1}} \subset B_{1}$.

Proposition 6.1. Suppose $f$ is a bounded continuous function on $F_{i_{1}}$. Suppose $v_{1}$ and $v_{2}$ are two bounded continuous functions on $V_{i_{1}}$, agreeing with $f$ on $F_{i_{1}}, C^{2}$ in $V_{i_{1}}-\{0\}$, satisfying $L v_{j}=0$ on $V_{i_{1}}-\{0\}, j=1,2$, and satisfying hypothesis $(2.8)(\mathrm{v})$ for some $R<1$. Then $v_{1}=v_{2}$ on $V_{i_{1}}$.

Proof. By considering $v=v_{1}-v_{2}$, we may suppose $f \equiv 0$. Since $L$ has smooth coefficients on $B_{1}-\{0\}$, if $v(0)=0$, then by the usual maximum principle, $v \equiv 0$ on $V_{i_{1}}$. We suppose then that $v(0) \neq 0$ and obtain a contradiction. By multiplying by a constant, we may suppose $v(0)=1$.

Consider $w(x)=P^{x}\left(\tau_{0}<\sigma_{i_{1}}\right)$. By Proposition 2.2, proved in $\S 3, v-w$ is bounded and continuous on $V_{i_{1}},(v-w)(0)=0, v-w=0$ on $F_{i_{1}}, v-w$ is $C^{2}$ on $V_{i_{1}}-\{0\}$, and $L(v-w)=0$ there. By the usual maximum principle, $v=w$ on $V_{i_{1}}$. 
If $x=(r, \theta) \in V_{i_{1}}$ is such that $u(x)=\beta$, then

$$
\begin{aligned}
w(x) & =P^{x}\left(\tau_{0}<\sigma_{i_{1}}\right)=P^{x}\left(u\left(X_{t}\right) \text { hits } 0 \text { before hitting } 2^{i_{1}}\right)=\frac{2^{i_{1}}-u(x)}{2^{i_{1}}} \\
& =1-\frac{\beta}{2^{i_{1}}}=1-c \beta=1-c u(x)=1-c r^{\alpha} d(\theta) .
\end{aligned}
$$

But then $D_{r r} w(x)=c|x|^{\alpha-2} d(\theta) \geq c|x|^{\alpha-2}$. And

$$
\int_{B_{R}} \sum_{i, j=1}^{d}\left|D_{i j} w\right|^{1+\eta}(x) \Gamma(x) d x \geq c \int_{0}^{R} r^{d-1}\left(r^{\alpha-2}\right)^{1+\eta} r^{2-\alpha-d} d r=\infty,
$$

since by Lemma 4.6, $\alpha \leq 1$, hence $d-1+(1+\eta)(\alpha-2)+2-\alpha-d<-1$. This contradicts the assertion that $v=w$ satisfied (2.8). Hence the proof is complete.

We now complete the proof of Theorem 2.3 in the case $\bar{\mu}<0$.

Theorem 6.2. Suppose $\eta>0$. There is at most one function $h$ satisfying hypotheses $(2.8)(\mathrm{i})-(\mathrm{v})$.

Proof. Just as in Proposition 6.1, the theorem will be proved if we show $w(x)=$ $P^{x}\left(\tau_{0}<\tau_{1}\right)$ does not satisfy $(2.8)(\mathrm{v})$.

Consider the Dirichlet problem on $V_{i_{1}}$ with boundary function $w$. Let $h(x)=E^{x} w\left(X_{\sigma_{i_{1}}}\right)$. If $r_{0}$ is taken small enough so that $B_{r_{0}} \subseteq V_{i_{1}}$, then by the strong Markov property $h(x)=E^{x} h\left(X_{\tau_{r_{0}}}\right)$. By applying scaling to Theorem $5.5, h$ satisfies $(2.8)(\mathrm{i})-(\mathrm{v})$ if $B_{1}$ is replaced by $B_{r_{0}}$. Also, since $h$ solves the Dirichlet problem on $V_{i_{1}}-B_{r_{0}}$ with boundary function $w$ on $F_{i_{1}}, h$ on $\partial B_{r_{0}}$, $h$ is one solution to the Dirichlet problem on $V_{i_{1}}$ satisfying the hypotheses of Proposition 6.1. Moreover, by Corollary 5.4, there exists $\delta>0$ such that

$$
\underset{B_{r}}{\operatorname{Osc}} h \leq c r^{\alpha+\delta} \text {. }
$$

Suppose now that $w(x)$ satisfies $(2.8)(\mathrm{v})$. By the proof of Proposition 2.2, $w$ also satisfies the hypotheses of Proposition 6.1. Therefore, by the conclusion of Proposition 6.1, wh on $V_{i_{1}}$.

But by Proposition 4.5, we know $1-w(x) \geq c|x|^{\alpha}$ for $x$ small. Since $w(0)=1$, this contradicts $(6.2)$. Therefore $w$ cannot satisfy $(2.8)(\mathrm{v})$, which proves the theorem.

\section{Polar, transient case}

In this section we prove Theorem 2.3 in the case $\bar{\mu}>0$. The proof is considerably easier in this case.

For $b>1$, define

$$
\begin{gathered}
Q_{b} f(x)=E^{b x}\left(f\left(X_{\tau_{1}}\right) ; \tau_{1}<\infty\right), \\
Q=Q_{2} .
\end{gathered}
$$


Completely analogously to the $\bar{\mu}<0$ case, $Q_{b}$ is strongly positive and compact. The eigenfunction $d(\theta)$ corresponding to the largest eigenvalue of $Q$ is strictly positive. As in $\S 4$, there exists $\alpha>0$ such that

$$
u(x)=E^{x}\left(d\left(X_{\tau_{1}}\right) ; \tau_{1}<\infty\right)=r^{-\alpha} d(\theta), \quad x=(r, \theta), r>1,
$$

is $C^{\infty}$ and solves $L u=0$ in $\bar{B}_{1}^{c}$, but now note the exponent of $r$ in (7.2) is negative. Arguing as in (4.6),

$$
-\alpha(-\alpha-1) d / 2-\alpha(2 \mu+1) d / 2+L_{S} d-\alpha M(r d)=0
$$

for all $\theta$, hence $L u(x)=0$ for all $x \neq 0$. Therefore $u\left(X_{t \wedge \tau_{0}}\right)=u\left(X_{t}\right)$ (since $\tau_{0}=\infty$, a.s.) is a local martingale.

Let

$$
V_{i}=\left\{x: u(x) \geq 2^{-i}\right\}, \quad i=\ldots,-2,-1,0,1,2, \ldots,
$$

so that we still have $V_{i} \subseteq V_{i+1}$. Define $F_{i}$ and $\sigma_{i}$ as in (4.8), (4.9).

Theorem 7.1. There exists a constant $c$ (depending on $\alpha$ ) such that

$$
\Gamma(x) \leq c|x|^{2-d} \quad \text { a.e. on } B_{1} .
$$

Proof. The proof is very similar to that of Theorem 4.7, with one exception. If $z \in F_{i+4}, i \leq i_{2}-5$,

$$
\begin{aligned}
P^{z}\left(\sigma_{i+3}<\sigma_{i_{2}}\right) & =P^{z}\left(u\left(X_{t}\right) \text { hits } 2^{-(i+3)} \text { before hitting } 2^{-i_{2}}\right) \\
& =\frac{2^{-(i+3)}-2^{-(i+4)}}{2^{-(i+3)}-2^{-i_{2}}} \leq 1-c, \quad c>0
\end{aligned}
$$

(cf. with $1-c 2^{i}$ in (4.16)). Just as in (4.17), $P^{x}\left(X_{T_{i}} \in F_{i+4}\right)$ is still bounded above and below by constants independent of $i$.

With (7.4), (4.19) becomes

$$
H_{i}^{\varepsilon} \leq c G_{i} \omega_{d} \varepsilon^{d} \leq c 2^{i(2-d) / \alpha} \omega_{d} \varepsilon^{d}
$$

and (4.20) becomes

which yields the desired result.

$$
\int_{B_{\varepsilon}(\eta)} \Gamma(z) d z \leq c|y|^{2-d} \omega_{d} \varepsilon^{d}
$$

Note that Proposition 5.3 makes no use of the hypothesis $\bar{\mu}<0$, and hence the assertion is valid in the cases $\bar{\mu} \geq 0$.

Suppose $f$ is bounded and continuous on $\partial B_{1}$. Let

$$
h(x)=E^{x} f\left(X_{\tau_{1}}\right) .
$$

Theorem 7.2. $h(x)$ defined by (7.6) satisfies (2.8)(i)-(v). There is at most one function $h$ satisfying $(2.8)(\mathrm{i})-(\mathrm{v})$.

Proof. Following the proof of Theorem 5.5, (2.8)(i)-(iv) hold. We may suppose $h(0)=0$ and $R<1 / 2$, and then exactly as in the derivation of $(5.10)$,

$$
\left|D_{i j} h(x)\right| \leq c|x|^{-2} \underset{B_{3|x| / 2}}{\operatorname{Osc}} h .
$$


Applying Proposition 5.3,

$$
\left|D_{i j} h(x)\right| \leq c|x|^{-2+\delta_{2}}\|f\|, \quad|x|<1 / 2 .
$$

Therefore, switching to polar coordinates,

$$
\int_{B_{P}}\left|D_{i j} h(x)\right|^{1+\eta} \Gamma(x) d x \leq c \int_{0}^{R} r^{d-1}\left(r^{-2+\delta_{2}}\right)^{1+\eta} r^{2-d} d r<\infty,
$$

provided $\eta<\delta_{2} /\left(2-\delta_{2}\right)$.

For the uniqueness assertion, apply the extended maximum principle Theorem 2.1 to the difference of any two functions $h_{1}, h_{2}$. Then $h_{1}-h_{2}=0$ on $B_{1}-\{0\}$, and by continuity, $h_{1}(0)=h_{2}(0)$.

Corollary 7.3. Corollary 5.6 holds in the cases $\bar{\mu} \geq 0$.

Proof. Use the estimate (7.8) and

$$
\left|D_{i} h(x)\right| \leq c|x|^{-1} \sup _{x \in B_{3|x| / 2}}|h(z)| \leq C|x|^{-1+\delta_{2}}
$$

in place of (5.10) and (5.11) in the proof of Corollary 5.6.

\section{Polar, Recurrent CASE}

It remains to prove Theorem 2.3 in the case $\bar{\mu}=0$.

Define $d$ and $u$ by (3.7) and (3.9). Let

$$
V_{i}=\left\{y=(r, \theta): r \leq 2^{i} e^{-d(\theta)}\right\}, \quad i=\ldots,-2,-1,0,1,2, \ldots,
$$

so that $V_{i} \subseteq V_{i+1}$, and if $y \in F_{i}=\partial V_{i}, u(y)=i \log 2$.

Theorem 8.1. There exists a constant $c$ such that

$$
\Gamma(x) \leq c(1+|\log x|)|x|^{2-d} \text { a.e. on } B_{1} .
$$

Proof. As in the proof of Theorem 7.1, we modify the proof of Theorem 4.7 by writing: if $z \in F_{i+4}, i \leq i_{2}-5$,

$$
\begin{aligned}
P^{z}\left(\sigma_{i+3}<\sigma_{i_{2}}\right) & =P^{z}\left(u\left(X_{t}\right) \text { hits }(i+3) \log 2 \text { before hitting } i_{2} \log 2\right) \\
& =\frac{i_{2}-(i+4)}{i_{2}-(i+3)} \leq 1-c(1+|i|)^{-1},
\end{aligned}
$$

leading to

$$
H_{i}^{\varepsilon} \leq c(1+|i|) G_{i} \omega_{d} \varepsilon^{d}
$$

and

$$
\int_{B_{\varepsilon}(y)} \Gamma(z) d z \leq c(1+|\log y|)|y|^{2-d} \omega_{d} \varepsilon^{d} .
$$

Let $f$ be bounded and continuous on $\partial B_{1}$,

$$
h(x)=E^{x} f\left(X_{\tau_{1}}\right) \text {. }
$$


Theorem 8.2. $h(x)$ defined by (8.5) satisfies (2.8)(i)-(v). There is at most one function $h$ satisfying $(2.8)(\mathrm{i})-(\mathrm{v})$.

Proof. The proof is identical to that of Theorem 7.2, except (7.9) becomes

$$
\int_{B_{R}}\left|D_{i j} h(x)\right|^{1+\eta} \Gamma(x) d x \leq c \int_{0}^{R} r^{d-1}\left(r^{-2+\delta_{2}}\right)^{1+\eta}|\log r| r^{2-d} d r<\infty,
$$

provided $\eta<\delta_{2} /\left(2-\delta_{2}\right)$.

Note that Corollary 7.3 includes the case $\bar{\mu}=0$ in its statement.

\section{REFERENCES}

1. R. F. Bass and E. Pardoux, Uniqueness for diffusions with piecewise constant coefficients, Probability Theory and Related Fields 76 (1987), 557-572.

2. J. R. Baxter and G. A. Brosamler, Energy and the law of the iterated logarithm, Math. Scand. 38 (1976), 115-136.

3. R. N. Bhattacharya, On the functional central limit theorem and the law of the iterated logarithm for Markov processes, Z. Wahrsch. Verw. Gebiete 60 (1982), 185-201.

4. D. Gilbarg and J. Serrin, On isolated singularities of solutions of second order elliptic differential equations, J. Analyse Math. 4 (1955/56), 309-340.

5. D. Gilbarg and N. S. Trudinger, Elliptic partial differential equations of second order, 2nd ed., Springer, New York, 1983.

6. M. G. Krein and M. A. Rutman, Linear operators leaving invariant a cone in a Banach space, Amer. Math. Soc. Transl. 10 (1962), 199-325.

7. N. V. Krylov, An inequality in the theory of stochastic integrals, Theory Probab. Appl. 16 (1971), 438-448.

8. N. V. Krylov and M. V. Safonov, An estimate of the probability that a diffusion process hits a set of positive measure, Soviet Math. Dokl. 20 (1979), 253-255.

9. A A certain property of solutions of parabolic equation with measurable coefficients, Math. USSR-Izv. 16 (1981), 151-235.

10. L. Lamberti and P. Manselli, Existence-uniqueness theorems and counterexamples for an axially symmetric elliptic operator, Boll. Un. Ital. B 2 (1983), 431-443.

11. C. Pucci, Limitazioni per soluzioni di equazioni ellittiche, Ann. Mat. Pura Appl. (4) 74 (1966), 15-30.

12. M. V. Safonov, Unimprovability of estimates of Hölder continuity for solution to linear elliptic equations with measurable coefficients, Mat. Sb. 132 (174) (1987), 275-288. (Russian)

13. D. W. Stroock and S. R. S. Varadhan, Multidimensional diffusion processes, Springer, New York, 1979.

14. R. J. Williams, Brownian motion with polar drift, Trans. Amer. Math. Soc. 292 (1985), 225-246.

15. L. Caffarelli, private communication.

Department of Mathematics, University of Washington, Seattle, Washington 98195 